\title{
Geometry Optimization of Sliding Inductive Links for Position-independent Wireless Power Transfer
}

\author{
Alex Pacini ${ }^{1}$, Franco Mastri ${ }^{1}$, Riccardo Trevisan ${ }^{1,2}$, Diego Masotti ${ }^{1}$, and Alessandra Costanzo ${ }^{1}$ \\ ${ }^{1}$ DEI - "Guglielmo Marconi”; School of Engineering and Architecture, University of Bologna, Italy \\ ${ }^{2}$ IMA SpA, Bologna, Italy
}

\begin{abstract}
In this paper we describe a geometry design solution to minimize performance variations of a wireless power transfer system "on the move" (WPT-Mob). A sequence of switchable couples of coils, connected in series or in parallel, is adopted at the fixed transmitting link side; the geometry of the moving receiver is optimized to keep the coupling factor, and thus the power transfer, constant during the movement. First the analytical formulation of the link coupling factor is derived as a function of its circuit-equivalent parameters computed by fullwave simulation. Then selected geometry parameters of the receiver side are optimized to keep the coupling factor constant while the link is moving. A set of TX-RX arrangements are simultaneously analysed to emulate the sliding movement of the WPT-Mob. The final optimized geometry demonstrates that a constant power transfer on-the-move is enabled, even for variable TX-to-RX link distances. Design and experimental verification are carried out for a geometry suitable for medium power transfer (tens of Watts) at $6.78 \mathrm{MHz}$, but the method is formulated in such a way that the system can be scaled up and down to accomplish different application needs.
\end{abstract}

Index Terms-Moving Vehicles, Near-Field WPT, coupling factor.

\section{INTRODUCTION}

Significant activity is devoted to minimize performance degradation of inductive wireless power transfer (WPT) systems when variable coils distance and/or misalignment are unavoidable [1], such as for moving objects [2,3] or implantable devices powering. However the conditions a WPT system with sliding coils has to fulfil, in order to maintain constant performance on the move, are still not completely assessed.

This work proposes a method to bridge this gap by cooptimizing the RX and TX coils geometries, in order to ensure constant coupling factor during the receiver displacement. A typical application for such a system is the power transfer from the pavement to a moving vehicle, where the coils can be placed underneath. Sliding WPT systems also have great potential in the industry [1]. Such sliding elements, or movers, act as small autonomous convoys included into some industrial equipment, such as a production line or an automatic machine. Movers, supplied by the coupled magnetic field, and controlled via an integrated wireless interface, are designed to transport certain items through pre-defined guided paths, selectively stop at particular stations along the path, and carry out some simple tasks by exploiting on-board manipulators. A schematic rendering of a wirelessly powered mover is shown in Fig. 1. The advantage of on-board energy availability is twofold: first to actuate the convoy by an electric motor along a mechanical guide; second, to perform tasks, such as item loading/unloading or item sealing, which are again actuated by on-board electrical work tools.

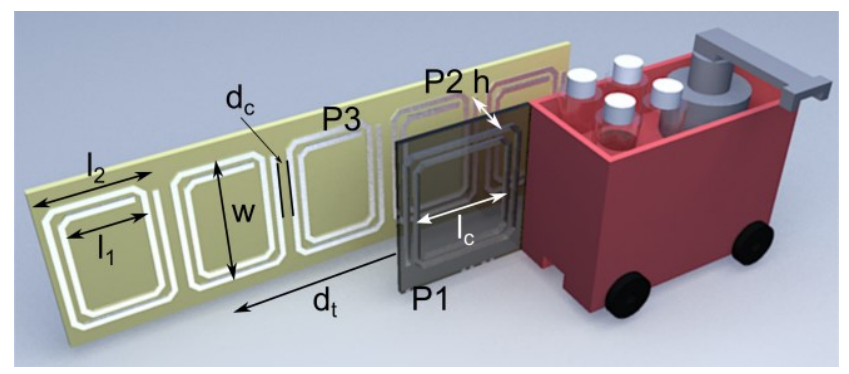

Fig. 1. Concept of a wirelessly powered mover, with two transmitters and one receiver involved at a time. $\mathrm{P}_{1}, \mathrm{P}_{2} \mathrm{P}_{3}$ are the WPT-link ports; $h$ is the TXto-RX distance, $w, l_{l}$ and $l_{2}$ are the TX-coil geometrical parameters: $d_{c}$ is the distance between two TX-coils; $l_{c}$ is the designable RX coil length. $d_{t}$ is the RX-to-TX displacement $\left(d_{t}=0 / d_{t M A X}\right.$ the $\mathrm{RX}$ center is aligned with the first/second TX-coil center). Simulations prototype dimensions (mm): $l_{1}=118$, $l_{2}=176, w=236, h=60$. The measures are done with a 6 times scaled version, as stated in the following.

Conceptually, movers are similar to moving vehicles models [2,3], or to sliding loads in [4]. Usually, to solve the problem of variable couplings, long primary current loops are adopted, which unavoidably introduce large primary leakage inductances, and consequently a significant drop of the overall power transfer efficiency [5].

As an alternative, in this paper we propose an optimized segmentation of the transmitters that allows a more energy efficient powering of the sliding path. By activating in the powering path two transmitting coils, connected in series, at a time, and by sliding the movable one over them, we demonstrate that an optimum choice of the RX and TX reciprocal coil lengths exists, enabling a constant coupling coefficient, and thus a constant power transfer and efficiency, during the RX coil translation. The results are experimentally demonstrated at $6.78 \mathrm{MHz}$, for few Watts power transfer.

A system implementation is proposed with a voltage-fed power source and an immittance inverter at the receiver side, to ensure a constant-amplitude output voltage. Extensive circuit simulation results are presented. A suitable switching network is designed to activate one couple of TX coils at a time, depending on the receiver position and its effects on the WPTMob operation is discussed. 


\section{WPT-MOB ANALYSIS AND DESIGN}

\section{A. Link geometry selection}

With reference to Fig. 1, let us consider the instantaneous three-port $\left(\mathrm{P}_{2}, \mathrm{P}_{3}, \mathrm{P}_{1}\right)$ WPT-link, consisting of two TX and one RX coils. The coplanar and fixed TX coils, 2 and 3, are placed in the pavement, while the RX-coil 1 slides in a parallel plane at distance $h$. The layout dimensions are intended for medium (few Watts) power transfer. The coils substrate is FR4.

For any varying position of the moving coil (starting from coil 1 being co-axial with coil $3, d_{i}=0$, up to coil 1 being in the midway between the coils 2 and 3 centres, $d_{t}=d_{t M A X} / 2$ ), the system coupling factor is computed by EM simulation. The situations for $d_{t M A X} / 2<d_{t}<d_{t M A X}$ can be derived by symmetry, since the TX coils are identical.

\section{B. Analytical design}

In a moving vehicle scenario, the varying $\mathrm{RX}$ coil position, with respect to the coupled TX ones, causes a significant oscillation of the coupling factor and thus a continuous variation of the transferred power. Two approaches are conceptually feasible to face this problem: i) a standard single coil active at a time [5]; ii) the solution we propose in this paper, with two (or possibly more) coils powered simultaneously.

In the first case, a long movable RX coil is used: each subsequent TX is turned on as the previous one is turned off, providing that the switching between them occurs in maximally flat coupling conditions. In this way, a moving coil long more than twice the fixed one is needed and a reduction of the coupling coefficient is unavoidable, while the flatness can be affected by the TX-RX distance $h$.

In this work we propose a new configuration with two coils active at a time, that are turned on during the time when the receiver is located above them. The two active coils can be connected in series or parallel and, since there is a nonnegligible coupling between the TX coils, due to the short distance $d_{C}$, the mutual influence between them needs be accounted for during the system optimization.

In order to determine the optimal RX coil geometry, we first compute the impedance matrix of the 3-port network of Fig. 1, by full-wave simulations at $6.78 \mathrm{MHz}$, and derive the coupling factor $k_{i j}$ between the coil pairs $(i, j=1, \ldots, 3)$ as follows:

$$
k_{i j}=\frac{\operatorname{Im}\left(Z_{i j}\right)}{\sqrt{\operatorname{Im}\left(Z_{i i}\right) \operatorname{Im}\left(Z_{j j}\right)}}
$$

After series or parallel connections of the two TX coils, a 2port network is obtained. Here we consider series connection; a comparison of the two choices can be found in [6]. The resulting inductance at the TX side is:

$$
L_{S}=2 L_{T X}\left(1+k_{23}\right) \text {, }
$$

which is lower than $2 L_{T X}$ since $k_{23}<0$, for the present geometrical configuration. The coupling coefficient $k_{S}$ of the final two-port link configuration can be derived as:

$$
k_{S}=\frac{\sqrt{2}}{2} \frac{k_{12}+k_{13}}{\sqrt{1+k_{23}}} .
$$

Using (2) and (3), the link mutual inductance $\mathrm{M}_{\mathrm{S}}$ can be derived as:

$$
M_{S}=k_{S} \sqrt{L_{S} L_{R X}},
$$

where $L_{R X}$ is the receiver inductance.

The optimisation of the TX and RX coils geometry is then performed by enforcing a constant value of $k_{S}$ for any possible reciprocal position of the coils.

\section{Validation by EM simulation and measurements}

Fig. 2 shows the coupling factor variations with respect to the TX and RX displacements, computed by (3) and using the EM simulation results, for the series connection of TX coils. The RX coil length, $l_{c}$, normalized with respect to the TX one, $l_{1}$, is used as the parameter.

These plots show that, when the RX-to-TX displacement varies as in Fig. 2, coupling factor variations can be as large as $30 \%$, if proper relative geometries of the TX and RX link sides are not selected. Indeed, if the optimum ones are adopted, a fixed coupling can be ensured for both series and parallel configurations [6]. For the present numerical example, with $d_{c}$ $=10 \mathrm{~mm}$, the optimum moving coil length normalized to the TX one is 1.43 (actually $l_{c}=169 \mathrm{~mm}$ ).

It is noteworthy that the order of magnitude of the chosen dimensions is suitable for the $6.78 \mathrm{MHz}$ operating frequency and for power range of the order of few tens of Watts, but it can be scaled up and down to be exploited for different application needs. Attention should be put in avoiding issues due to parasitic effects, thus the operating frequency and the associated geometry should be chosen in such a way that the coils operate far from their parasitic resonance.

Given the optimum geometries, the dependence of the link behaviour on distance is investigated in Fig. 3: these plots show that the adopted design choices also guarantee a constant power transfer at different link distances $(h)$. The set-up for the experimental validation is shown in Fig. 4, where the Sparameters of the resulting two-port link are measured. One couple of fixed transmitting coils is used to emulate the instantaneous picture of the WPT-Mob operation.

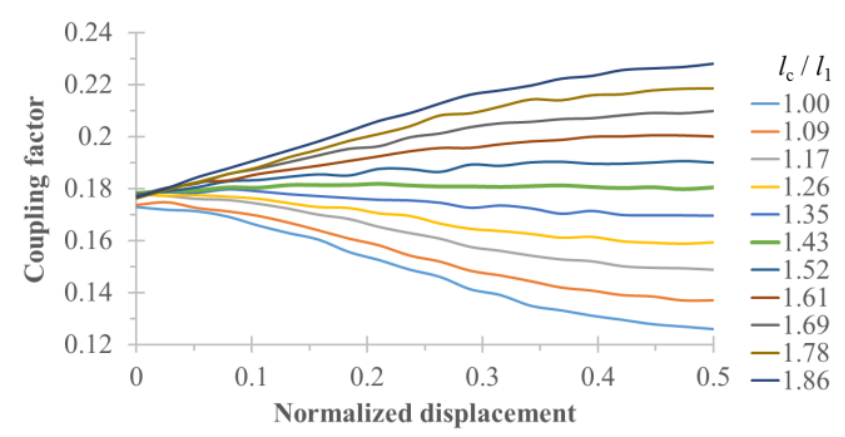

Fig. 2. Coupling factor between moving RX and fixed TX coils connected in series as a function of the normalized displacement $d_{t}$. The parameter is the RX coil length normalized to the TX one.(see Fig. 1) 


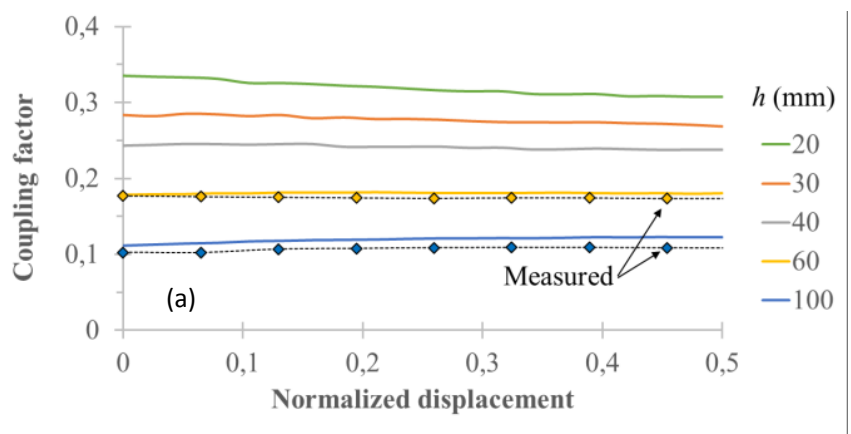

Fig. 3. Measured and predicted coupling factor between the moving and the series-connected fixed coils, as a function of the normalized displacement $d_{t}$ for different link distance $(h)$.

Taking advantage of the full scalability of the proposed approach, all the coils geometries are reduced by a factor of 6 , with respect to the example of application used to demonstrate the analytical procedure. Two distances of about $10 \mathrm{~mm}$ and 17 $\mathrm{mm}$ are tested and the measured results are superimposed in Fig. 3. Excellent agreement with the predicted behaviour is observed in both cases.

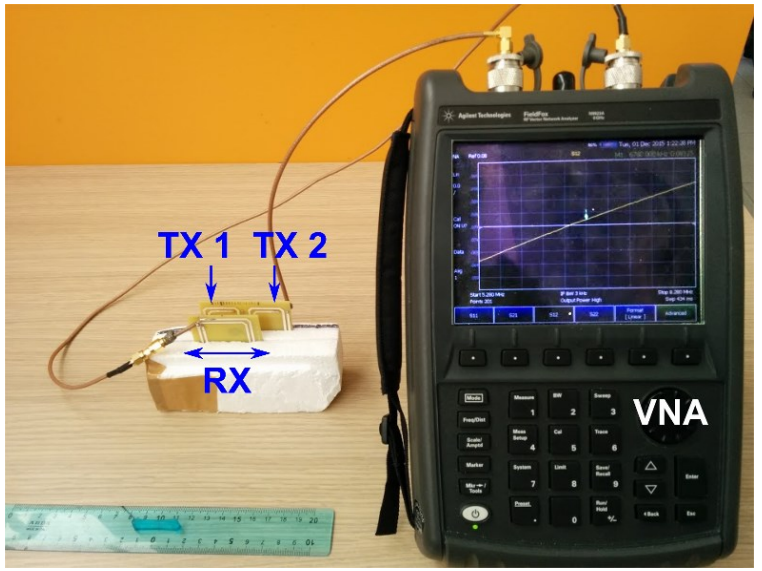

Fig. 4. Photo of the mesurement setup: a Fieldfox handheld VNA is used to measure the two-port S-parameters of the WPT-mob link, consisiting of two series-connected transmitting coils and one receiving coil with optimized geometries.

\section{CIRCUIT SIMULATION OF A POSSIBLE WPT-MOB IMPLEMENTATION}

Given the optimized wireless link, the transmitter and receiver active circuits and the associated control network are designed. Let us assume that the system is driven by a voltage source $V_{G}$. In order to obtain an output voltage practically independent of the load resistance, the WPT link should ideally behave as a transformer [7]. This result can be achieved by the circuit implementation shown in Fig. 5, where the mutual coupling between the TX and RX inductors is represented by the T-network formed by $-M_{S}, M_{S},-M_{S}$. The inductance $L_{S}$ is compensated by adding a series capacitor $C_{S}$ resonating at the link operating frequency. A parallel capacitor $C_{R X}$, resonating at the same frequency, and a series inductor $L_{R X}^{\prime}$, equal to $L_{R X}$, are connected on the receiver side. At the operating frequency, the T-network $\mathrm{L}_{\mathrm{RX}}, \mathrm{C}_{\mathrm{RX}}, \mathrm{L}_{\mathrm{RX}}^{\prime}$ behaves as an immittance inverter, hence the whole network acts as two cascaded immittance inverters, which are equivalent to an ideal transformer [7].

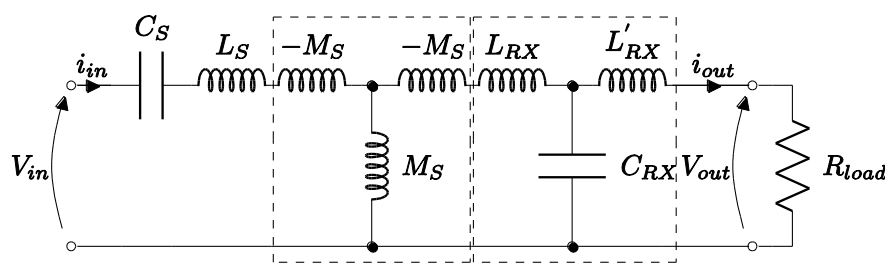

Fig. 5. Circuit equivalent of the WPT-Mod implementation to ensure a an output voltage $\mathrm{V}_{\text {OUT }}$ independent of the load.

Fig. 6 shows the circuit description of an instantaneous WPT-Mob system configuration: the upper part represents the transmitter circuit at the time when the mobile receiver (lower part) is located between the first and the second TX coils. It has to be noticed that the capacitance $C_{S}$ of Fig. 5 is split in Fig. 6 into two capacitances $C_{T X}$ connected in series to two consecutive TX inductors. The switches $S W_{U 1}, S W_{S 2}, S W_{S 3}$, $S W_{D 4}$ are closed while all the others are open.

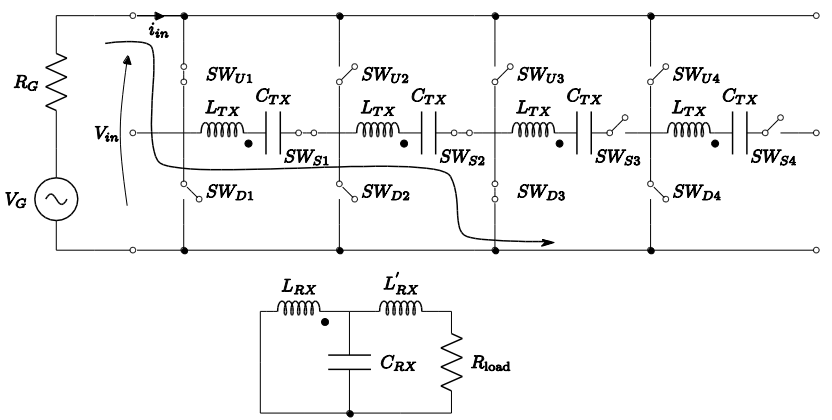

Fig. 6. Circuit representation of the entire WPT-Mob link showing the current path when the RX coil is located between the first two TX coils. The system is modular and can be replied by repeating the cell.

The performance of this system implementation are summarized in Fig. 7, where the computed output voltage, for several TX-to-RX relative positions, as in Fig. 2, are plotted as a function of the load resistance: the curves are almost superimposed to one onother and this confirms that the chosen design approach ensures output voltage variations lower than $1.2 \%$ for any TX-to-RF reciprocal position. Furthermore, an almost constant output voltage in a wide range of loading conditions can be also observed, which is a primary requirement of the proposed system architecture. The corresponding power transfer efficiency is also shown on the same plot.

As the receiver proceeds further, an optimal switches configuration at the TX side has to be configured, in order to avoid abrupt interruptions of the current paths. To solve this problem, the intermediate circuit configuration, shown in Fig. 8 , is adopted, where the current paths are highlighted. 


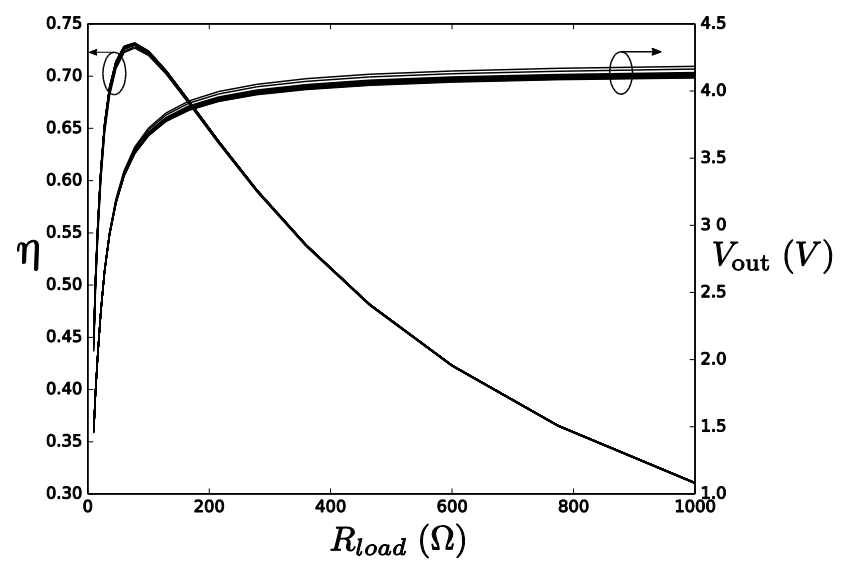

Fig. 7. Predicted output voltage and power transfer efficiency of the WPTMob configured as in Fig. 6. The plots parameter is the receiver dispalacement $d_{t}$. The data are for the 6 times scaled version with an $h=60 \mathrm{~mm}$ (thus $10 \mathrm{~mm}$ when scaled).

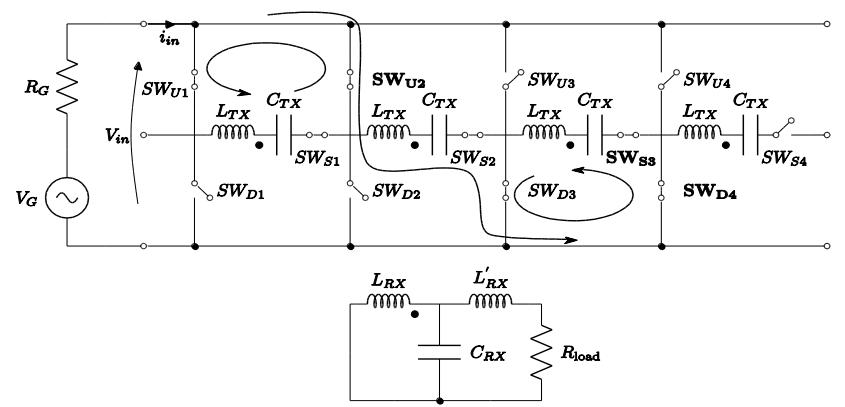

Fig. 8. Proposed switches intermediate state. $S W_{D 4}, S W_{S 3}, S W_{D 2}$ are closed, two potentially critical current closed loops are created.

It must be noted that the closed loops in Fig. 8 create high-Q resonators, which could cause a signficant degradation of the system efficiency if their resonant frequency were equal to the link operating frequency. However, since the equivalent series inductance $L_{S}$ is less then $2 L_{T X}$, their resonant frequency is slightly lower than the link resonant frequency and the flat behaviour of the efficiency can be preserved.

The predicted operation of the intemediate state is shown in Fig. 9, where the link efficiency is plotted as a function of the operating frequency and of the load resistance. A deep valley can be observed in correspondence of the closed loops resonant frequencies.

\section{CONCLUSIONS}

A new approach for achieving constant coupling in moving resonant WPT systems has been proposed. Referring to electrical vehicle applications, a series of TX coils are disposed along the path and a couple of them are simultaneously activated while the vehicle is moving over, obtaining a practically flat coupling coefficient. Each time the RX coil aligns with one of the TX coils, the preceding coil is deactivated while the following is activated. The system behaviour is thus periodic and the coupling coefficient can be kept constant along all the vehicle path, thus making easier the fulfilment of conditions such as the maximum power transfer.
The proposed design method obtains a flat coupling factor even with different coil distances.

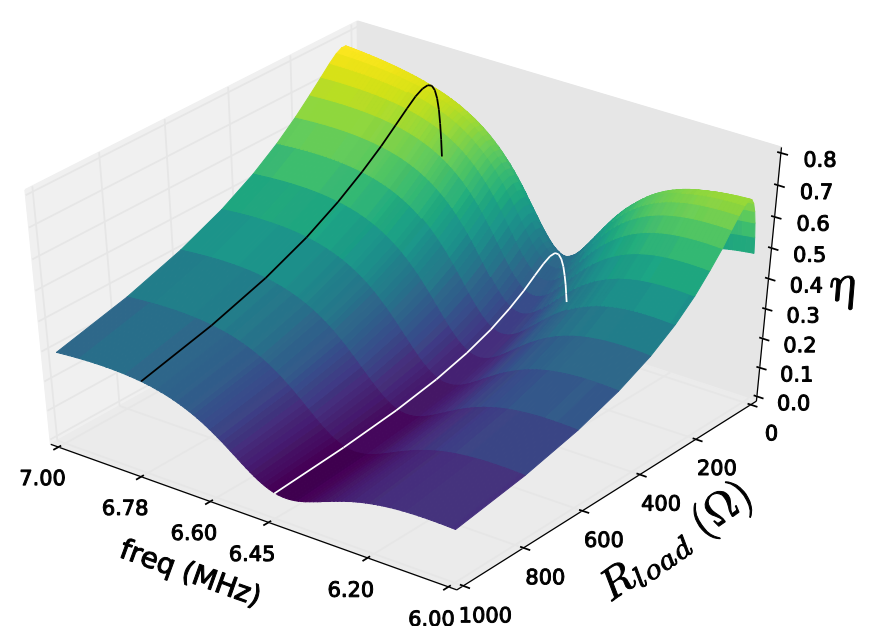

Fig. 9. Effects of the intermediate switching case: the notch of the efficiency, due to high losses in the closed loops, occurs at a frequency (indicated by the white line) which is different from the link main resonanting frequency (indicated by the black line).

\section{ACKNOWLEDGMENT}

This work was partly supported by the EU Artemis JTI project: "Arrowhead" and by a research contract granted from IMA SpA.

\section{REFERENCES}

[1] L.J. Chen, J.T. Boys, G.A. Covic, "Power Management for MultiplePickup IPT Systems in Materials Handling Applications," IEEE Trans. Emerg. Sel. Topics Power Electron., vol. 3, no. 1, pp. 163-176, Mar. 2015.

[2] Chao-Shui Lin, Sheng-Guo Lin, Ching-Feng Chang, Hsun-Hung Li, Liang-Rui Chen, "Model of contactless power transfer system for linear track," Proc. IEEE 2009 Int. Conf. Power Electronics and Drive Systems, pp. 1075-1079, Taipei, Nov. 2009.

[3] Jia-You Lee, Hung-Yu Shen, Kai-Chuan Chan, "Design and Implementation of Removable and Closed-Shape Dual-Ring Pickup for Contactless Linear Inductive Power Track System," IEEE Trans. Ind. Appl., vol. 50, no. 6, pp. 4036-4046, Nov./Dec. 2014.

[4] F.F.A. van der Pijl, M. Castilla, P. Bauer, "Adaptive Sliding-Mode Control for a Multiple-User Inductive Power Transfer System Without Need for Communication," IEEE Trans. Ind. Electron, vol. 60, no. 1, pp. 271-279, Jan. 2013

[5] J. P. C. Smeets, T. T. Overboom, J. W. Jansen, E. A. Lomonova, "Comparison of Position-Independent Contactless Energy Transfer Systems," IEEE Trans. Power Electron., vol. 28, no. 4, pp. 2059-2067, Apr. 2013.

[6] A. Pacini, F. Mastri, R. Trevisan, A. Costanzo and D. Masotti, "Theoretical and experimental characterization of moving wireless power transfer systems," 2016 10th European Conf. on Antennas and Propagation, (Davos, Switzerland), Apr. 2016.

[7] A. Costanzo, M. Dionigi, F. Mastri, M. Mongiardo, J. A. Russer, P. Russer, "Rigorous Network Modeling of Magnetic-Resonant Wireless Power Transfer" Cambridge Journal on Wireless Power Transfer, Vol. 1, No. 1, March 2014, pp. 27-34. 